\title{
Globalización y confianza: información no-financiera
}

\author{
Oscar Banda Lefaure \\ Director comercial de PricewaterhouseCoopers \\ Profesor del Departamento Académico de Administración de la Universidad del Pacífico
}

\begin{abstract}
Resumen
Los cambios en la forma de hacer negocios que se han propiciado a partir de la globalización de los mercados han permitido un sinnúmero de beneficios, pero también un número importante de riesgos, que se comprobaron cuando a partir del año 2001 se sucedieron uno tras otro los destapes de escándalos financieros en todas partes del mundo. Estos lamentables hechos pusieron al descubierto la vulnerabilidad a la que están expuestos los inversionistas (y demás stakeholders) por no contar con información oportuna, clara y precisa de la marcha de la empresa en la que invierten, razón por la cual no pueden tomar precauciones. Además, estas catástrofes empresariales fueron muestra de cómo los ejecutivos más inescrupulosos no dudan en actuar de manera ilegal para ocultar sus turbias maniobras financieras y contables, con el fin de propiciar su beneficio personal. Subyace entonces la política de compensación de ejecutivos. Ante esta coyuntura se empieza a hablar con más fuerza de la importancia de migrar hacia un nuevo modelo de divulgación, donde la comunidad financiera global pueda tomar refugio de estas malas prácticas y confiar en los controles que el directorio y la gerencia establecen. Este nuevo modelo de divulgación tiene uno de sus pilares en los reportes de información no-financiera. Esta no es una solución alquimista, sino que es uno de los muchos esfuerzos que deben emprender las empresas para recuperar la confianza dañada por otros. La crisis financiera internacional que afecta a la economía mundial en estos momentos es otro ejemplo de la necesidad de dotar de mayor confianza a los stakeholders -mediante la transparencia en la información que se les provea-. Solo así se podrá mantener e incrementar su participación en el mercado de capitales y reducir, además, los sobrecostos que trae consigo la falta de confianza generalizada en que vivimos.
\end{abstract}

\section{Palabras clave}

Modelos de divulgación, Reportes corporativos, Confianza, no-financiera

\section{Introducción}

«Gracias a las fuerzas que ha desatado la globalización en los últimos tiempos, las estructuras económicas, comerciales y políticas están pasando de ser rígidamente verticales a ser francamente horizontales; es así como el mundo se está "aplanando“"»

(Friedman, Milton. El mundo es plano, 2005).
En la expresión «el mundo es plano», debe entenderse plano como sinónimo de conectado. La disminución de barreras comerciales y políticas, y el exponencial crecimiento de los avances técnicos y tecnológicos de la revolución digital, han hecho posible hacer negocios y llegar a acuerdos de manera instantánea con miles de millones de 
personas a lo largo y ancho del planeta. Esta es la conclusión a la que arriba Friedman en relación con la globalización.

Junto con la globalización llegaron tantas nuevas oportunidades como retos en los más diversos sentidos. Una de dichas oportunidades es el acceso global a las fuentes de capital. Así, cualquier inversionista (sea una persona natural o jurídica), independientemente de su ubicación física, puede invertir su dinero en cualquier empresa sin importar dónde esta se halle domiciliada, el giro de negocio, el uso horario, el idioma, la moneda, etcétera.

Este milagro del entorno económico actual debe ser un círculo virtuoso por el cual, tanto los inversionistas como las empresas, logren una mejora de su bienestar económico. Sin embargo, existe un reto implícito que condiciona la calidad virtuosa del proceso: la confianza recíproca entre ambos. En esta, como en cualquier relación humana, la confianza es una piedra angular que se ha deteriorado, aun más cuando en este mundo plano se requiere de una dosis adicional de confianza porque las partes involucradas se desenvuelven -muchas veces- en contextos totalmente distintos (país, huso horario, moneda, idioma, etcétera.). No pensemos solo en las inversiones directas -cuando ' $A$ ' invierte en ' $B$ '-, donde se ha generado una confianza entre ambos. Pensemos, en cambio, en los escenarios en donde ' $B$ ' invierte en ' $C$ ', este en ' $D$ ' y así sucesivamente. Al final, sin proponérselo, ' $A$ ' termina depositando su inversión en ' $D$ ' y es posible que ni lo sepa. Los recientes sucesos de bancarrotas y reestructuraciones que se han vivido en el mundo son una muestra de ello, ya que la esencia plana del mundo actual hace que las empresas estén muchas veces entrelazadas en el nivel de sus inversiones.

El deterioro de la confianza, mencionado líneas arriba, se ha producido por un sinnúmero de eventos lamentables, algunos de los cuales serán revisados a continuación.
Objetivo

El presente documento busca profundizar en el uso y beneficio de las informaciones no financieras como parte de la estrategia de divulgación de las empresas.

\section{Metodología}

Se analizará la secuencia de hechos que nos han traído a este punto de desconfianza y se revisará las alternativas que se tienen a mano. Finalmente, un breve análisis de la situación local nos permitirá tomar el pulso a nuestro entorno más cercano.

\section{Marco conceptual}

Las informaciones no financieras son más una corriente que una teoría, razón por la cual no existe un marco conceptual teórico definido. Por ello, el presente documento recoge y desarrolla los aportes de distintas fuentes y autores, lo cual representa, en conjunto y de manera estructurada, la mejor aproximación a una base teórica para la presente investigación.

Los detonantes y los paliativos

Por lamentable coincidencia, junto con la llegada del nuevo milenio fuimos testigos de catástrofes y escándalos financieros que dispararon alertas al más alto nivel dentro de la comunidad financiera global y empresarial, pues se puso de manifiesto la capacidad que tienen las empresas (personificadas por inescrupulosos empleados en todo nivel) de solapar sus malas prácticas dentro de la información financiera que se creía un reflejo fiel de la realidad y que fomentaba en los inversionistas a una falsa sensación de tranquilidad sobre su capital invertido. Del mismo modo, se evidenció cómo el marco de divulgación tradicional era vulnerable ante las prácticas menos éticas nunca antes mostradas a la luz. Son muchos los ejemplos que presenciamos casi simultáneamente. 
Uno de los primeros y sin duda el más emblemático fue el de Enron, donde las políticas de compensación de ejecutivos «fomentaron» en ellos una avaricia desmedida que los llevó a desarrollar «creativas» formas de incrementar las ventas, las que lindaban con lo anti-ético y luego generaron una bola de nieve tal, que -para evitar que los aplaste- los indujo a prácticas criminales como la falsificación de registros, la destrucción de documentos e incluso el recorte de la energía en todo un estado, con el fin de justificar el incremento de tarifas eléctricas y generar así mayores ingresos. Las consecuencias de este escándalo son ya conocidas: 20.000 empleados perdieron sus empleos y seguros de vida, así como sus fondos de retiro, que ascendían a US $\$ 1.200$ millones en total. Los ex trabajadores perdieron US\$ 2.000 millones en fondos de pensiones. En el nivel judicial, la triste reseña incluye: 15 declaraciones de culpabilidad y 6 condenas, sin contar los procesos que se siguieron a empleados de otras empresas vinculadas al escándalo.

Otro caso muy conocido fue el de una importante empresa tecnológica en el año 2002, cuando sus ejecutivos no dudaron en registrar contratos de arrendamiento como ventas y así provocar un crecimiento artificial en las ventas, lo que generó una diferencia de US\$3.000 millones, quizá motivados también por los jugosos réditos de sus bonos. «No inventaron dinero que no existía, lo tomaron prestado del futuro». Sin embargo, estas calamidades no fueron exclusividad de la primera economía mundial (Estados Unidos). Casi en paralelo, en Italia salía a la luz las fraudulentas actividades de un importante conglomerado de productos lácteos, cuyas repercusiones alcanzaron a su filial en Argentina. En este caso se trató de una sucesión de fallas en los controles internos y externos de la compañía, que permitieron el desvío de fondos para ayudar a otras sociedades del grupo que atravesaban una crisis financiera, para lo cual no hubo escrúpulo que impidiera incluso la falsificación de documentos.

Retomando el enunciado inicial, en un mundo plano, del mismo modo en que todos estamos conectados para lo favorable, lo estamos también para lo adverso. Por ello, estos eventos trascendieron a todas partes del planeta. En un mundo globalizado, la pena y la gloria se comparten.

Es en este contexto donde surge la necesidad de implementar mayor control en la calidad de la información que los inversionistas reciben de las empresas. El factor de transparencia asociado al recurso información tomó desde entonces un carácter vital.

Uno de los efectos y consecuencias más tangibles fue la elaboración de la llamada Ley Sarbanes Oxley, cuyo título oficial en inglés es SarbanesOxley Act of 2002, Pub. L. No. 107-204, 116 Stat. 745 (30 de julio de 2002). Esta es una ley de los Estados Unidos también conocida como el «Acta de Reforma de la Contabilidad Pública de Empresas y de Protección al Inversionista», SOx o SarbOx, que nace con el fin de monitorear a las empresas que cotizan en bolsa, y evitar fraudes y riesgo de bancarrota, protegiendo de esta manera al inversionista. Esta ley, más allá del ámbito nacional, afecta a todas las empresas que cotizan en la NYSE (Bolsa de Valores de Nueva York), así como a sus subsidiarias.

Al igual que el ejemplo de SOx, casi todos los países elaboraron regulaciones complementarias con el mismo fin. Como es lógico, y en paralelo, los demás participantes de la comunidad financiera y los participantes del flujo de información (administradores corporativos, el consejo de administración, los auditores, los asesores y consultores externos, las agencias calificadoras de riesgo, entidades creadoras de principios contables, autoridades regulatorias, analistas independientes, instituciones financieras, entre otros) tuvieron que modificar sus prácticas para cubrir este vacío que había salido a luz. Sin embargo, el actor principal de esta obra debe seguir siendo la empresa, personificada en la gerencia. Son ellos los responsables de la preparación, producción y comunicación de la información que recibe la comunidad financiera; en ellos recae la mayor responsabilidad respecto de la calidad de la in- 
formación revelada. Por lo tanto, más que en pensar en controles y paliativos debemos pensar en un cambio integral que parta de la empresa, para que el mercado retome el nivel de confianza que tenía antes de las debacles financieras mencionadas anteriormente. En términos generales el flujo de la información dentro de los mercados de capitales se resume en el Gráfico 1.

\section{El papel que desempeña la contabilidad}

La contabilidad desempeña un papel preponderante, pues es el medio de comunicación natural entre empresas e inversionistas en el nivel de información financiera. Es común que los lectores de los estados financieros midan la salud de la empresa a partir de la evaluación de dicha información. Sin embargo, esto no es tan cierto, porque la contabilidad es una ciencia de registro y cálculo donde la buena fe y la transparencia de la gerencia al elaborar la información son premisas esenciales. Un punto muy importante es que el modelo de información financiera tradicional satisfizo plenamente las expectativas del mercado de capitales en sus inicios, ya que se trataba de un entorno más sencillo, donde los activos eran tangibles, los ingresos y los costos relativamente fáciles de definir y calcular, y los pasivos se podían medir con mayor claridad y certeza. Con el tiempo, los mercados cambiaron, se tornaron más complejos, mientras el modelo tradicional no se alteró mayormente en su esencia.

Siendo el vehículo de comunicación más estandarizado, no debe llamar nuestra atención que haya sido en torno a la contabilidad donde se hayan desarrollado tantas modificaciones en lo que respecta a las normativas contables y regulatorias. El principal aspecto modificado ha sido, sin duda, el nivel de revelación y la calidad de la evidencia que sustente la información que la empresa revela.

\section{Gráfico 1}

Flujo informativo en los mercados de capitales

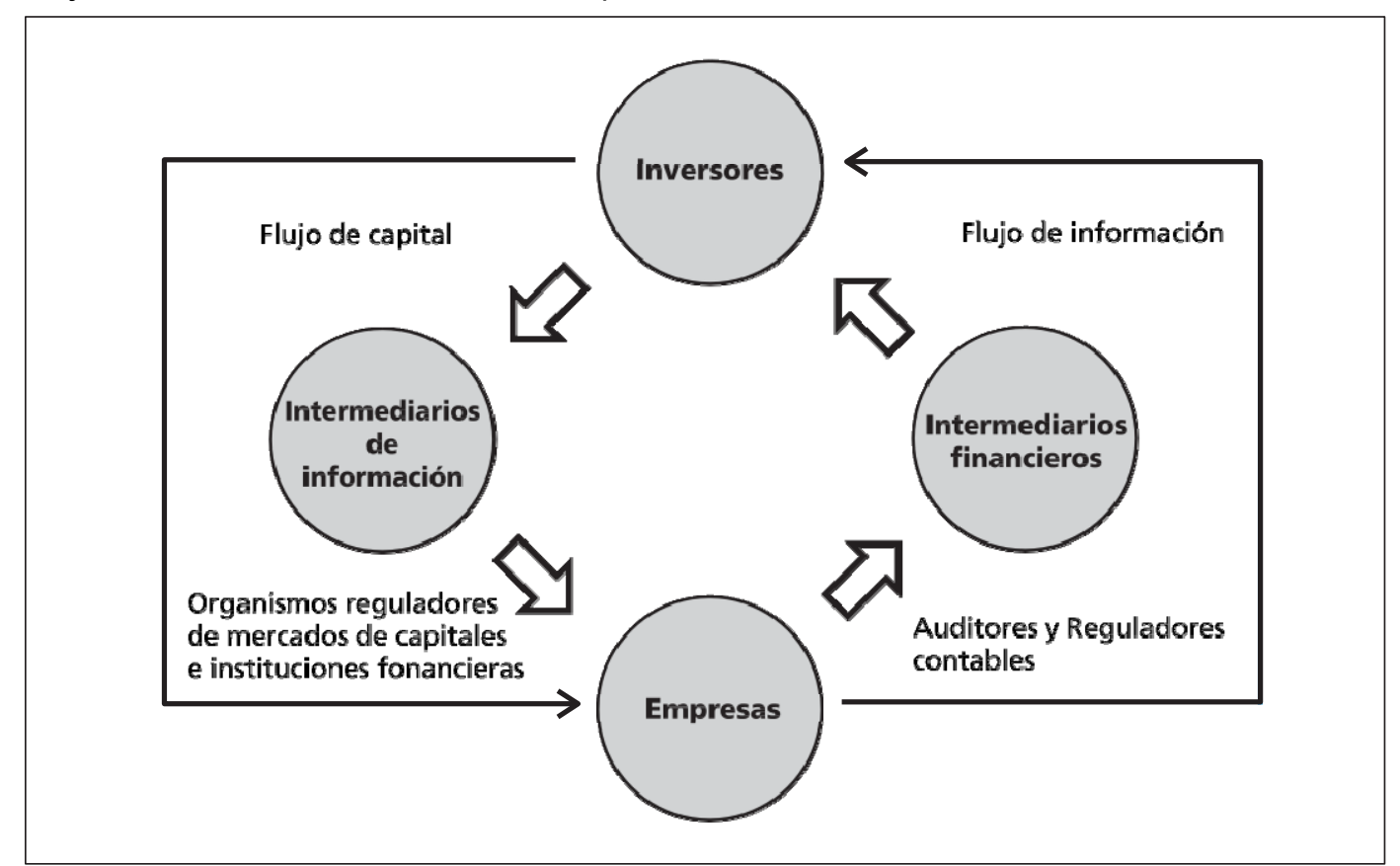

Fuente: Healey, P.M. y Palepu, K.G. (2001): «Information asymmetry, corporate disclosure, and the capital markets: a review of the empirical disclosure literature», journal of Accounting and Economics, vol. 31, pp. 405-440. 
Pese a lo anterior, aún persiste una sensación de vulnerabilidad en la comunidad financiera porque no se ha tocado el problema de fondo: la modernización del modelo de divulgación de información corporativa. El modelo actual es objetado por concentrase en la forma legal de la transacciones más que en el valor económico, donde prima el pasado sobre el futuro, donde el costo tiene más importancia que la generación de valor y donde el concepto de beneficio está sobre el de riqueza (The Institute of Chartered Accountants of Scotland). Ya en 1995, Steve Wallman -Ex comisario de la Securities and Exchange Commission (SEC)- afirmaba que: «la información financiera no está adaptándose al cambio en los negocios».

Un paliativo insuficiente ha sido el complemento que brindan las agencias de rating, analistas financieros externos y agencias especializadas, quienes elaboran y divulgan información complementaria que, aunque preparan con la mejor intención y mayor capacidad, no llega a ser comparable con la información proporcionada directamente por la empresa, en tanto esta es la única que tiene la fuente directa para la elaboración de cualquier reporte

\section{La esperanza: información no financiera}

Ante la pérdida de relevancia de la información financiera, la comunidad financiera global tuvo que buscar una nueva fuente de información que satisfaga los requerimientos, ahora más exigentes, del mercado. La respuesta la encontraron en reportes que, por ejemplo, eran utilizados por los directivos para la gestión interna, como el MD\&A (Management's Discussion and Analysis o «Análisis y discusiones de la gerencia»), el Estado de valor agregado, el Informe de sostenibilidad, etcétera. Si bien estos reportes pueden clasificarse en distintos tipos, tienen un objetivo común: capturar no solo el valor actual de los activos, sino sobre todo su habilidad para producir valor en el futuro.

Revisemos, brevemente, algunos de estos reportes mencionados como ejemplo. El MD\&A es un reporte que se incluye, por lo general, como parte del reporte anual de la empresa, donde la gerencia comparte aspectos importantes vinculados al pasado y presente de la compañía. Es común que incluya una revisión de las operaciones del último año y se compartan las perspectivas de la gerencia para el año entrante, así como sus metas y cursos de acción definidos para alcanzarlas. Lamentablemente, en términos generales, el MD\&A, en nuestros mercados, es preparado solo ante una coyuntura de exposición (cuando se busca inversionistas, ante una oferta pública de acciones, etcétera), luego de lo cual se retorna al modelo tradicional. Por su parte, el Estado de valor agregado muestra el total de riqueza generada y cómo esta se distribuye entre empleados, accionistas, proveedores financieros, gobierno, etcétera; e incluye también cuánto será retenido por la empresa. Este reporte asume como premisa que el valor agregado es la diferencia entre el costo de los insumos/recursos y el precio de venta de los bienes/ servicios. El Reporte de sos-tenibilidad es un documento que muestra las actividades realizadas por la empresa para asegurar su sostenibilidad en el tiempo, cuidando especialmente el medio ambiente en el que se desarrolla y la sociedad con la que se vincula. Este es quizá el tipo de reporte más amplio y utilizado de los aquí mencionados-, pues las actividades desarrolladas con el objetivo de asegurar su sostenibilidad en el largo plazo pueden ser entendidas bajo muchos puntos de vista y dimensiones. Para evitarlo existen estándares, como los del Global Reporting Initiative (GRI) o los del Instituto Ethos del Brasil, que detallan los aspectos sobre los cuales la empresa debe pronunciarse y comentar su nivel de evolución.

Nuestro entorno es una clara muestra de cómo los factores sociales y/o ambientales pueden constituir un riesgo muy grave para las empresas y llegar a comprometer al accionista, ya que al descuidarlo amenazan la continuidad de su negocio y, por tanto, las eficiencias conseguidas en cualquier área operativa o administrativa dejan de tener relevancia cuando se producen, por ejem- 
plo, bloqueos, huelgas, enfrentamientos, etcétera. Estas situaciones son muy importantes para las operaciones vinculadas a actividades extractivas, dado que la comunidad tiene el sentido de posesión especial de los recursos que se extraen del lugar donde ellos viven. Sin embargo, no se limita a estas industrias porque los temas de calentamiento global e impacto al ecosistema pueden asociarse a cualquier actividad que se emprenda.

Como afirma Richard Baker (citado por Porto 2005: 40): «la contabilidad global ha llegado». Al respecto, Nelida Porto (Profesora de la Universidad de Santiago de Compostela) afirma que «transformará la manera en que las empresas informarán sobre sus beneficios (al ser actualmente dos organismos FASB e IASB los que dominan el panorama armonizador), entonces, la armonización será la tendencia a seguir por la información no financiera» (Porto 2005: 40).

\section{El objetivo de las informaciones no financieras}

El objetivo de cualquier medida no financiera debería ser capturar los datos que informan sobre aquellos aspectos del negocio que no estén reflejados en los estados financieros básicos (Upton 2001).

Entre las diferentes ventajas que se asocian a los indicadores o informaciones no financieras destacan su relevancia, ya que permiten mostrar de manera aislada el comportamiento de cada una de las variables importantes, sin agregaciones que dificulten la detección de problemas; su oportunidad, puesto que no requieren el cierre del ciclo contable; su capacidad de motivación, porque reflejan mejor el nivel de productividad y eficacia e inducen con más facilidad a la toma de decisiones; y su relevancia con respecto al ámbito temporal de las decisiones, habida cuenta de que son fundamentales para garantizar la rentabilidad de la empresa en el largo plazo (Larrán y García-Meca 2004).
Una de las críticas más comunes a las informaciones no financieras es que al ser de revelación voluntaria y, por tanto, muy pocas veces auditadas, en ocasiones resultan en confesiones de parte, donde las empresas revelan lo que les favorece y omiten los aspectos que no les son favorables. Por consiguiente, pueden subyacer potenciales riesgos. En vista de ello, es importante que se desarrollen modelos y guías de reporte que estandaricen estas informaciones; luego, una auditoría podrá brindar una opinión imparcial sobre la veracidad y certeza de la información revelada. Con una base estándar y auditada se podrá tener total confianza sobre la información recibida y los inversionistas podrán tomar sus decisiones con cada vez mejor información.

Dos criterios importantes respecto de las informaciones no financieras son: el aspecto voluntario y la estrategia de revelación de la empresa. El primero queda tácito al no ser, por lo pronto, información exigida por los reguladores, por lo que su preparación y divulgación es un derecho pleno de la empresa. En términos de Meek et al. (1995: 555): "Voluntary disclosures are the focus of an increasing amount of attention by accounting researchers. Voluntary disclosures -disclosures in excess of requirements- represent free choices on the part of company managements to provide accounting and other information deemed relevant to the decision needs of users of their annual reports». En ese sentido, podemos ver ejemplos de empresas que realizan y hacen público un reporte solo en los años en que el resultado las beneficia, y omiten su preparación y/o divulgación cuando no le es favorable, de manera que el inversionista no puede trazar una línea de evolución en el tiempo. Aquí es donde los participantes de la comunidad financiera desempeñamos un papel vital, en nosotros recae la responsabilidad de motivar -y debería decir exigir-a las empresas para que mantengan una cultura continua de divulgación voluntaria de información adicional a la financiera. Debemos también incentivar el uso de estándares para la preparación de dichos reportes, con una guía homogénea, con el fin que no resulte en una declaración de parte y 
que las empresas más comprometidas con la transparencia en la divulgación de la información propicien la verificación de dichos reportes por firmas externas.

Un inhibidor común, que suelen mencionar las empresas que no revelan más allá de la información financiera obligatoria, es el factor estratégico/competitivo. En su opinión, las informaciones no financieras exponen la estrategia de la compañía y, por tanto, la convierten en vulnerable ante sus competidores, lo que representaría una desventaja para el inversionista por el riesgo potencial de este perjuicio. Es por ello crucial que la gerencia defina con cuidado qué información puede revelar, que resulte provechosa para sus stakeholders, sin hacerse vulnerable frente a sus competidores y que, al mismo tiempo, cumpla con los estándares internacionales para que satisfaga los principios de relevancia, oportunidad y transparencia que persigue como objetivo este tipo de reportes. Sin duda, hay un gran número de informaciones estratégicas e indicadores que pueden ser revelados sin poner en riesgo la situación de la empresa.

Finalmente, la cura parece estar muy cerca de la causa de la enfermedad. Este problema se originó por la pérdida de confianza del inversionista en cuanto a la información proporcionada por la em- presa. Por lo tanto, es razonable pensar que sea esta la que tome las riendas de la solución y diseñe un modelo de revelación que satisfaga las expectativas de los inversionistas y recuperen así, poco a poco, su confianza. Subyace además un objetivo sumamente tangible, ya que la desconfianza fue aplacada con controles que incrementaron los costos en todos los sentidos (personal dedicado a realizar controles, mayor tiempo en preparar la información, mayores costos de revisión por parte de los reguladores, mayor burocracia-más tiempo en detrimento de la operación, mayor profundidad en la auditoría, más detalle en la documentación, etcétera), por lo que este puede ser uno de los pocos ejemplos donde la confianza tiene un precio objetivo. Sería cándido esperar que este proceso se lleve a cabo únicamente por deseo de la empresa, por lo que los participantes de la comunidad financiera global debemos redoblar esfuerzos para fomentar esta transición para el beneficio de todos.

\section{Análisis del entorno local}

Se analizó la información no financiera que presentan las empresas en nuestro entorno, para lo cual se tomó como muestra las empresas que componen el Índice Selectivo de la Bolsa de Valores de Lima (IS-BVL) a diciembre de 2008 (véase el gráfico 2). Los resultados obtenidos mues-

Gráfico 2

Divulgación de información no financiera en el Perú, IS-BVL 2008

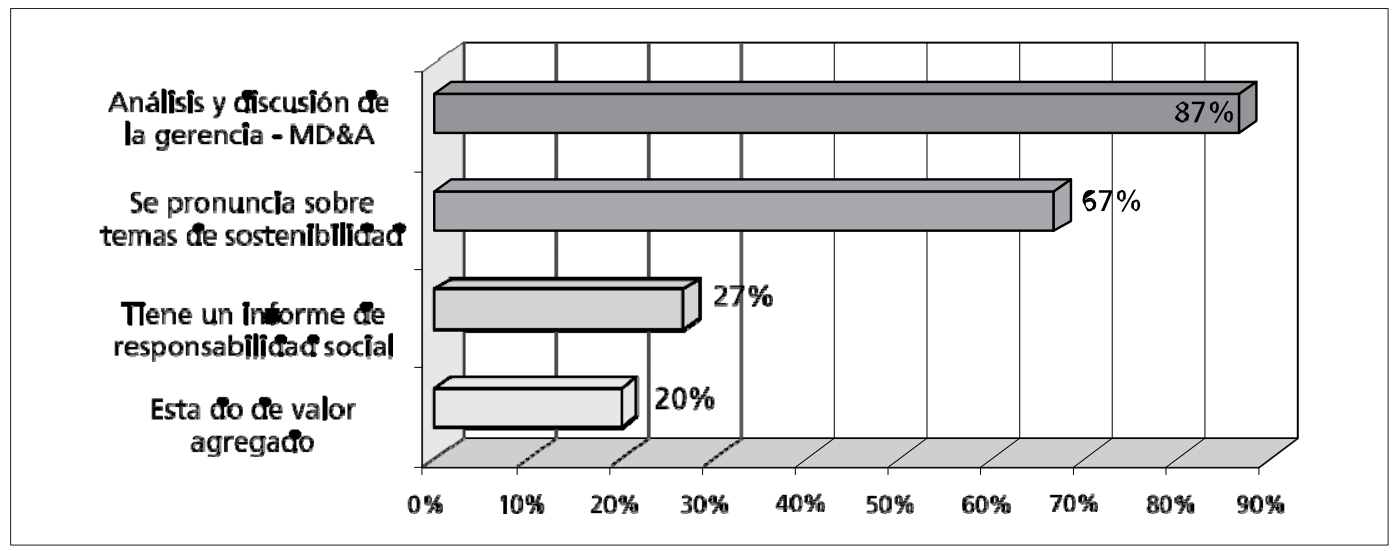

Elaboración propia. 
tran que cerca del $90 \%$ de las 15 empresas que componen el IS-BVL, hacen públicos sus reportes de MD\&A. Se aprecia también que hay un $67 \%$ de empresas que se pronuncia respecto de temas de sostenibilidad; sin embargo, solo el $40 \%$ de ellas (4 empresas) elabora un informe de sostenibilidad que se acerca a los estándares mundiales. Dichos «pronunciamientos» son, en su mayoría, confesiones de parte que las empresas divulgan, abordando de manera superficial los temas muchas veces circunscritos, única y exclusivamente, al ámbito de la responsabilidad social. Respecto del Estado de valor agregado, se debe señalar que solo el $20 \%$ de las empresas manifestó elaborar este reporte y solo una lo tenía a disposición del público en su página web. En economías más avanzadas es insólito encontrar que siquiera una de las empresas que componen los principales índices bursátiles no cuente con esta información abierta al público.

Es importante resaltar el papel de la Comisión Nacional Supervisora de Empresas y Valores (Conasev). A ella se debe, en gran medida, el alto porcentaje de presentación del «Análisis y Discusión de la Gerencia» (MD\&A), ya que todas las empresas emisoras de valores están obligadas a reportarla trimestralmente. La presentación de esta información está debidamente reglamentada en el Manual para la preparación de información financiera, en cuya sección tercera se detalla:

«10.002. Análisis y Discusión de la Gerencia El análisis y discusión se basa en los estados financieros intermedios, describe y explica las principales características de la situación financiera de la empresa, su desempeño financiero y los principales riesgos e incertidumbres que ésta enfrenta.

Consiste en un comentario analítico de la gerencia, respecto del resultado de las operaciones y de la situación económica financiera de la empresa, cuyo fin es facilitar el análisis y la comprensión de los cambios importantes que hayan ocurrido correspondientes al último período y su variación respecto al mismo período correspondiente al año precedente y de ser relevante respecto al cierre del período anterior, así como de las tendencias y posibles estrategias a seguir en el futuro. La discusión debe enfocarse en el negocio total y en sus principales subdivisiones.

Los comentarios deberán permitir un mejor entendimiento de la situación revelada en los estados financieros y no limitarse a mencionar cuánto crecieron o decrecieron determinadas cuentas, sino que se deberá exponer las razones de estas variaciones.

Asimismo, la discusión y el análisis deben dar a conocer información de las transacciones y otros eventos inciertos que la gerencia estima podrían causar que los estados financieros presentados no sean necesariamente indicativos de los resultados económicos futuros o de la futura posición financiera. Se puede señalar eventos que han tenido relevancia en el pasado y que no se espera que la tengan en el futuro y eventos que no hayan tenido relevancia en el pasado y que se espera que la tengan en el futuro. De esta manera, la discusión no debe quedar reducida a una simple repetición de las cifras mostradas en los estados financieros de períodos intermedios» (Conasev s.f.: 67).

Pese a no ser un tema que tenga carácter de mandato, algunas empresas presentan ante la Conasev información complementaria de manera voluntaria, como es el caso del Informe de sostenibilidad, lo que denota una voluntad de transparencia muy meritoria.

Adicionalmente, es oportuno precisar que dentro de la información que los emisores de valores presentan a la Conasev, se puede encontrar algunos de los contenidos que llamamos informaciones no financieras. Así, por ejemplo, parte de la información contenida en el Estado de valor agregado se expone en las notas a los estados financieros relativas a: costo de ventas, otros costos operacionales, gastos de ventas y gastos de ad- 
ministración. Esto se ha establecido en el Manual para la preparación de información financiera (numerales: $7.200,7.201,7.250,7.300 \mathrm{y}$ 7.310) (Conasev s.f.: 57-58). Respecto de la responsabilidad social, la Conasev exige su exposición en la nota a los estados financieros como 'Efectos ambientales por las actividades de la empresa'.

Este nivel de revelación observado entre los emisores de valores, a solicitud de la Conasev, amerita una reflexión adicional en cuanto al nivel de detalle, homogeneidad de información para fines comparativos, difusión y carácter voluntario de la revelación. Estos cuatro conceptos están unidos detrás de una estrategia de revelación de información no financiera. Si bien es una práctica sana que la información se revele dentro del paquete que se reporta ante la Conasev, el modelo por seguir es aquel en el que la empresa se pronuncia voluntaria y consistentemente sobre la base de estándares de revelación en el tema (añadiendo cálculos o reseñas comparativas año a año cuando sea posible) y con un nivel de detalle que provea al lector de un nivel de confianza adicional al que tenía. El cuarto punto se relaciona con la difusión. Actualmente, la información no financiera que los emisores presentan a la Conasev se encuentra en el portal de Internet de esta entidad. Sin embargo, esta debería añadirse a la información corporativa dentro del portal de la propia empresa y, en la medida de lo posible, difundir esta y cualquier publicación. Así, el interesado podrá encontrar con mayor facilidad la información complementaria sobre la compañía sin recurrir a otras fuentes, como son los distintos reguladores gubernamentales. La práctica de difusión considera que toda la información no financiera que se decida revelar sea preparada y distribuida con el mismo empeño que con el que se realizan y distribuyen las memorias, los estados financieros, etcétera.

\section{Conclusión}

Es preocupante que al analizar la situación de quince de las empresas más representativas de nuestro entorno, se encuentre tan bajo nivel de adaptación de los nuevos vientos que soplan en cuanto a la divulgación de información no financiera. Si bien es una muestra muy pequeña, su representatividad nos hace inferir que la situación en el resto de empresas debe ser peor. Se debe tener en cuenta que la información no financiera tiene en su esencia una búsqueda del incremento de confianza y, en esa dirección, los más interesados en generar confianza deberían ser las empresas públicas (entendidas como aquellas que cotizan en bolsa). Aunque nuestra bolsa de valores no tiene los niveles de sofisticación que quisiéramos, sería razonable esperar que al menos los integrantes de los principales índices tengan el máximo nivel de transparencia en la información que revelan. A manera de sugerencia, se podría incluir la revelación de información no financiera dentro de los principios de buen gobierno corporativo, que son evaluados por la Bolsa de Valores de Lima con el fin de decidir la incorporación de las empresas calificadas dentro del índice del mismo nombre. Actualmente, el «evaluador» considera 26 principios, dentro de los cuales se hace referencia al tema del presente documento en dos de ellos (Conasev 2005):

«9. Principio IV.D.3).- Los casos de duda sobre el carácter confidencial de la información solicitada por los accionistas o por los grupos de interés relacionados con la sociedad deben ser resueltos. Los criterios deben ser adoptados por el Directorio y ratificados por la Junta General, así como incluidos en el estatuto o reglamento interno de la sociedad. En todo caso la revelación de información no debe poner en peligro la posición competitiva de la empresa ni ser susceptible de afectar el normal desarrollo de las actividades de la misma.; [y]

17. El Directorio debe realizar ciertas funciones claves, a saber:

Principio (V.D.7).- [que dice solamente] Supervisar la política de información».

Al respecto, resultaría beneficioso incluir la revelación de información no financiera como un fac- 
tor a evaluarse en términos de detalle, homogeneidad, difusión, carácter voluntario, etcétera.

Es necesario reflexionar si en las empresas públicas el nivel de revelación de información no financiera con fines de transparencia corporativa tiene o no resultados similares a los observados en las empresas privadas. Resulta razonable esperar que, lamentablemente, el nivel de revelación sea incluso menor. Esto debe ser motivo de preocupación, ya que pese a la evolución de nuestra economía aún necesitamos de mucho esfuerzo y compromiso para alcanzar niveles internacionales, especialmente en los mercados financieros.
Finalmente, en medio de la coyuntura actual de crisis internacional, surgen voces autorizadas que comentan que aquellas empresas que han mostrado como práctica habitual la revelación voluntaria de información no financiera, han logrado generar un nivel de confianza en sus stakeholders que les ha permitido ver menos afectada su posición financiera. Estaremos atentos en observar si el tiempo confirma esta tendencia, que resultaría un aliciente adicional para demostrar las ventajas que trae consigo una estrategia de revelación de este tipo c. 
ABOODY, D. y R. KASZNIK

2000 «CEO Stock Options Awards and the Timing of Corporate Voluntary Disclosures». Journal of Accounting and Economics, vol. 29, $\mathrm{N}^{\circ}$ 21 , pp. $73-100$.

\section{ARELLANO, J.}

1999 «Indicadores de Gestión no financieros: cuáles, cuándo y para quién». X Congreso AECA. Zaragoza, setiembre.

\section{ARVIDSSON, S.}

2003 Demand and Supply of Information on Intangibles - The Case of Knowledge Intensive Companies. Estocolomo: Lund Business Press.

BEAVER, W. H.

1989 Financial Accounting: An Accounting Revolution. Englewood Cliffs, Nueva Jersey: Prentice Hall International.

BRANCATO, K. C.

1995 New Corporate Performance Measures: A Research Report. Nueva York: The Conference Board.

\section{CANADIAN INSTITUTE OF CHARTERED ACCOUNTANTS \\ 2000 «Total Value Creation». $<$ http://www.totalvaluecreation.com>.}

\section{COMISIÓN ALDAMA}

2003 Informe de la Comisión Especial para el fomento de la transparencia y seguridad de los mercados financieros y sociedades cotizadas. Nebrija, enero <http://www.nebrija.com/ nebrija-santander-responsabilidad-social/documentos/Informe_Aldama.pdf>.

COMISIÓN NACIONAL SUPERVISORA DE EMPRESAS Y VALORES (Conasev)

s.f. Manual para la preparación de información financiera. Lima: Conasev. Disponible en <http://www.conasev.gob.pe/acer cade/ ConsultaCiudadana/manual_Inf_Financiera.pdf>.
2005 Resolución Gerencia General NN 140-2005EF/94.11. Lima: 28 de diciembre.

\section{ECCLES, $R$}

1991 «The Performance Measurement Manifesto». En: Harvard Business Review, vol. 69, № 1 , pp. 131-137.

\section{ELLIOT, R. K. y P. D. JACOBSON}

1994 «Cost and benefits of business Information Disclosure». En: Accounting Horizons, vol. 8, No 4 , pp. 80-96.

\section{GARCÍA-MECA, E. e I. PARRA}

2003 «Las presentaciones a analistas financieros como canal de divulgación de información; evidencia empírica en el mercado de valores español». XII Congreso Aeca «Transparencia Empresarial y Sociedad del Conocimiento». Cádiz, setiembre-octubre.

GINER, B.

1995 La divulgación de información financiera: una investigación empírica. España: Ministerio de Economía.

\section{GINER, B. y A. MORA}

2002 «Información cualitativa: su papel en el proceso de toma de decisiones». VIII Jornada de Trabajo sobre Análisis Contable. Valladolid, setiembre.

GINER, B.; M. ARCE, N. CERVERA y A. RUIZ

2003 «Incentivos para la divulgación voluntaria de información: evidencia empírica sobre la información segmentada». En: Revista Europea de Dirección y Economía de la Empresa, vol. $12, N^{\circ} 4$, pp. $69-86$.

\section{GOLDBERG, R.}

2002 «Revelación de Información y Transparencia: Revelación de Información No-Financiera». The Third Meeting of the Latin American Corporate Governance Roundtable. México, abril. 
LARRÁN, M.

2001 El mercado de información voluntaria en las bolsas de valores. Madrid: Instituto de Contabilidad y Auditoría de Cuentas.

LARRÁN, M. y GARCÍA-MECA, E.

2005 «Costes, beneficios y factores ligados a la política de divulgación de información financiera». En: Revista de Contabilidad, vol. 7, No 14, pp. 75-112.

2004 «La relevancia de la información no financiera en la estrategia empresarial de divulgación voluntaria: percepciones empresa analista sobre su utilidad». Revista Valenciana de Economía y Hacienda, № 12 , pp. 128145.

MEEK, G.; C. B. ROBERTS y S. J. GRAY

1995 «Factors Influencing Voluntary Annual Report Disclosures by U.S., U.K. and Continental European Multinational Corporations». En: Journal of International Business Studies, vol. 26 , №3, pp. 555-572.

NUEVA ZELANDA, OFFICE OF THE CONTROLLER AND AUDITOR-GENERAL

2008 «Conceptual framework for reporting nonfinancial performance information» (Parte 6). En: The Auditors General's observations on the quality of performance reporting. Wellington, Nueva Zelanda: Office of the Auditor-General, pp. 37-49. <http:// www.oag.govt.nz/2008/per for mancereporting/docs/performance-reporting.pdf>.

PORTO, N.

2005 «Tendencias en la información no financiera para la gestión de la empresa socialmente responsable». En: Revista Iberoamericana de Contabilidad de Gestión, Nº 5, pp. 13-44.
PRICEWATERHOUSECOOPERS (PwC)

2008 Informaciones no financieras. Un nuevo modelo de divulgación. Colección PwC, 1. Lima: PwC. <http://www.pwc.com/pe/spa/pdf/ Col_01_InfNoFin.pdf>.

ROGERS, R. K. y J. GRANT

1997 "Content Analysis of Information Cited in Reports of Sell-side Financial Analysts». The Journal of Financial Statement Analysis, vol. 3, № 1, pp. 17-30.

\section{SCHIFF, A. D. y L. R. HOFFMAN}

1996 «An exploration of the use of financial and nonfinancial measures of performance by executives in a service organization». En: Behavioral Research in Accounting, vol. 8, pp. 134-154.

THE INSTITUTE OF CHARTERED ACCOUNTANTS OF SCOTLAND (ICAS)

2001 Making Corporate Reports Valuable, Editado por McMonnies, Edinburgh.

THE CANADIAN INSTITUTE OF CHARTERED ACCOUNTANTS

2004 Management's Discussion and Analysis: Guidance on Preparation and Disclosure. Canadá: The Canadian Institute of Chartered Accountants.

UPTON, W. S., Jr.

2001 Business and Financial Reporting. Challenges from the New Economy. Special Report $N^{\circ}$ 219-A. Norwalk, Connecticut: Financial Accounting Standards Board.

ZADEK, S. y P. RAYNARD

2004 The Future of Sustainability Assurance. ACCA Research Report N ${ }^{\circ}$ 86. Londres: Certified Accountants Educational Trus. 